\title{
A Short Range Wireless Communication Using Android NFC
} API

\author{
Durga Priya ${ }^{1}$, E.Ramakotaiah ${ }^{2}$ \\ '(CSE, Indur Institute of Engineering and Technology, India) \\ ${ }_{2}^{2}$ (CSE, Indur Institute of Engineering and Technology, India)
}

\begin{abstract}
In this paper, we are proposing the implementation of short range wireless communication using Android's NFC API. Near Field Communication is set of protocols used for communication between two android powered and NFC enabled devices. Near Field Communication is based on the Radio Frequency Identification standards used for contactless communication within the range of $4 \mathrm{~cm}$. There are two types of communications possible in NFC based services. One is Passive and another is Active communication, two unprogrammed NFC tags can be read in passive communication and in active communication both the devices diffuse power and supports peer to peer communication.
\end{abstract}

Keywords: Wireless communication, Near Field Communication, Android, NFC tag.

\section{Introduction}

Near Field Communication is one of implementation of ubiquitous computing in recent times. NFC system is designed from the collaboration of many technologies like wireless communications, mobile devices, mobile applications, and smart card technology and cloud services. NFC ecosystem is combination of contactless identification and interconnected technologies. NFC works between two devices within short range through touching paradigm. NFC works with mobile device on one side and NFC tag or another NFC enabled mobile device on other side.

The main idea of NFC is the integration of personal and sensitive information such as credit card into mobile phones. Because of the storage of private information security is the major concern and even the short distances supported by the RFID technology is considered as too long.

NFC works by performing handshake between two devices that are in close proximity. NFC allows for a simple data exchange between two devices by a way of physical touch. NFC requires an initiator and a target, the initiator produces a radio frequency field within a range of $4 \mathrm{~cm}$. The target picks up the RF field and receives the data it contains.

NFC is not a new technology, think of ID cards used at offices to open the doors, and also when the Master card implemented the "pay on the go" booths. NFC operates in three modes like Reader/Writer Mode, Peer-to-to Peer mode and card emulation modes. The reader/writer mode enables NFC enabled devices to exchange data with NFC tags. The Peer-to-Peer mode enables two NFC enabled mobile devices to exchange data. In card emulation mode, the NFC enabled mobile device acts as a smart card or contactless card.

NFC technology has its applications in various areas like Mobility, Access \& Transportation, Gaming, Loyalty \& Payments and Identification. Making payments with a touch at anywhere contactless card readers are deployed. Sharing business cards with other NFC enabled phones with just one touch. Taking a picture and transferring to NFC enabled printer and so on are some of the application areas of NFC technology.

This paper presents a mobile application that tracks the employee's transportation activity from office to home and from home to office. This is a useful application for tracking the employee going in cabs during night shift.

\section{Background/Related work}

There are wide variety of wireless communication technologies existing before the NFC technology. Some of them are RFID technology, Bluetooth and IRDA technologies. The major benefit of wireless communication is mobility which supports productivity and flexibility

RFID technology is used for exchanging data between an RFID reader and an RFID tag through radio waves. The RFID tags are generally attached to an object mostly for the purpose of tracking and identification. The below figure shows the RFID system and its components 


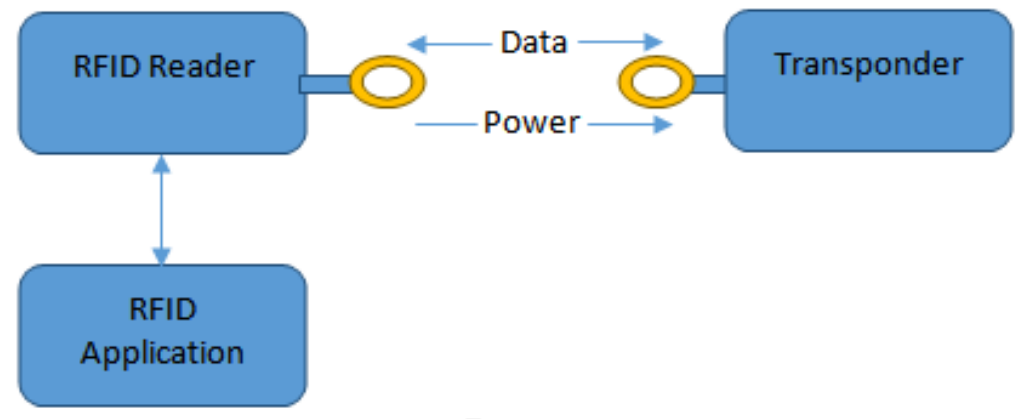

Figure 1- RFID System

In order to establish connection between RFID reader and the RFID application a wired or wireless networks can be used in different sections of communication. RFID tags contain an antenna and Integrated circuit(IC). An integrated circuit stores and processes the data, modulates and demodulates radio frequency signals and performs several other functions. Antenna receives and transmits the signal.

RFID system consists of two main components: the Transponder and Reader. Transponder is a component that is located on a product or an object to be identified and the reader is the one which reads from the transponder or writes to the transponder. The transponder contains a coupling element and an IC that carries the data to be transferred. Transponder is generally a RFID tag that can store large amount of data. There are two types of tags one is Passive tags which don't have power supply and the other type is active tagswhich have their own power supply. The Reader component contains a transceiver with a decoder for interpreting data, control unit and antenna.

RFID technology has many application areas like Inventory tracking, human implants, animal identification, casino chip tracking and hospital operating rooms and etc.

NFC technology is better when compared to the existing technologies in terms of setup time required for activation, usability and the customer experience. The shorter range of NFC enables us to develop personalized and customized applications. NFC technology supports range of operation up to $4 \mathrm{~cm}$ whereas RFID supports the range of operation up to $3 \mathrm{mts}$. RFID is basically used for tracking applications where NFC technology can be used for Payment related, peer-to-peer communication and etc. The data transfer rate of NFC is slower than the Bluetooth technology but faster than the infrared technology.

\section{Proposed System}

In our proposed system, we are developing an application to track the employee's transportation activity from home to office and from office to home. In order to execute the application, each employee needs an NFC tag that will be programmed to contain the ID of that employee. It is assumed that inside the cab, there is a NFC enabled mobile phone with the driver that can read the tags of the employee. When the employee gets into the cab, they touch their tags to the mobile device and the mobile saves the getting on time. When that employee gets off from the cab, they again touch their tag to the mobile and the mobile saves the getting off time. The in-time and off-time can be saved to the server online so that the employee family can see the information via a mobile application that is integrated to the system.

The above use case scenario is the example application of Reader/ Writer operation mode of NFC technology. NFC technology uses three smart devices as stated below:

- NFC-Enabled Mobile Phone: Integration of NFC technology with mobile phones creates a lot of opportunities for the ease of use.

- $\quad$ NFC Reader: This is capable of transferring data to another component. The example for this is contactless point of sale (POS) terminal.

- $\quad$ NFC tag: It is an RFID tag with no integrated power.

The Reader/Writer mode is about the communication of NFC enabled mobile device with an NFC tag for both reading and writing data from and to the tags. Below figure discusses the usage model of reader/writer operation mode. 


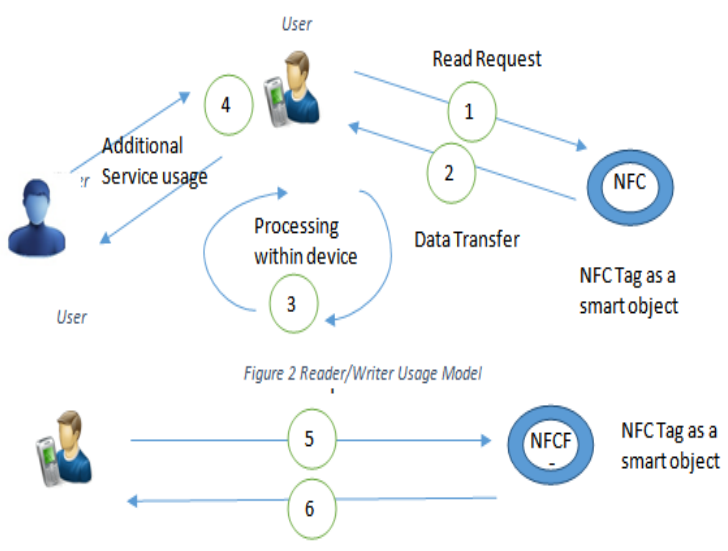

Acknowledgement

1. The first step is Read Request where the user sends a request for data by touching the mobile to NFC tag.

2. In the step 2 the data that exist in in the tag is transferred to the mobile device.

3. Third step deals with the data processing within the mobile device such as displaying data to the user

4. Fourth step performs any additional processing within the mobile like performing operations via internet connectivity and so on

5. Fifth step is where the user requests to write the data to NFC tag by bringing mobile phone in contact with the tag.

6. The NFC tag acknowledges the user about the success of the write operation.

We need to write two applications for this use case one is Writer Application and the other one is Reader application. The writer application includes an activity to write the employee's data to the tags. We have to write the NDEF message to the tag, when the tag is discovered in the proximity of the mobile device. We have to use android.nfc package to read and write the NDEF messages from and to the supported tags. Below is the activity flow figure of the application.

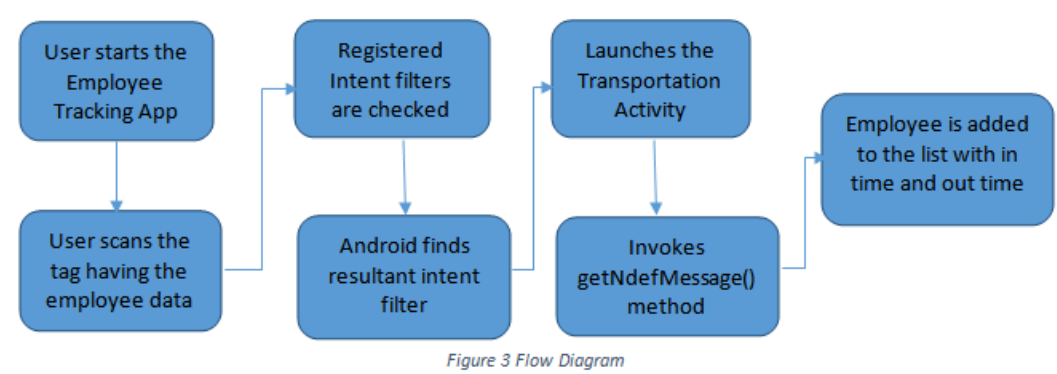

Below is the screenshot of the Employee Tracking application screens

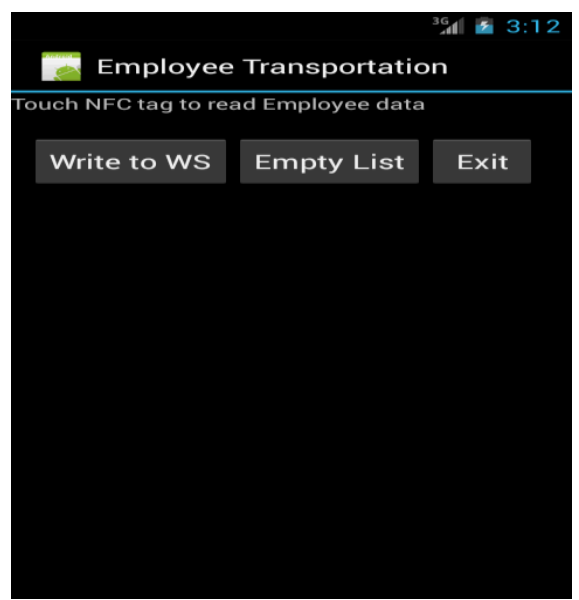




\section{Conclusion}

In the age of smartphones, many mundane services like ticketing, payment, tracking and etc. can be performed by the NFC enabled mobile phones. The market for the NFC enabled devices is massive. Presently there are one billion smart cards and four billion mobiles phones being used across the world [1]. It is estimated that by 2014 one in every six mobile phone users will have NFC enabled device [2]. NFC will also impact a wide spectrum of enterprises like transportation, healthcare, telecom and etc. This paper presents one use cases implementation of NFC in the Transportation domain.

\section{Future Scope}

The near future holds a bundle of smart applications that gain from Near Field Communication and their personalized applications. There are some challenges like data security in terms of eavesdropping, data modification, data corruption and relay attack. When the mobile phone is lost or change all the payment related data and applications have to transferred from one phone to another. There are security challenges involved in NFC technology which needs to be addressed in order to ensure secure transfer of data between devices.

\section{References}

[1] Infographic: Mobile statistics, stats \& facts 2011. Available at http://www.digitalbuzzblog.com/2011-mobile-statistics-stats-factsmarketing-infographic/.

[2] Levitt J.(2011), 1 in 6 mobile subscribers to have NFC mobile phones by 2014 according to Juniper research.

[3] Ortiz, C. Enrique "An Introduction to Near-FieldCommunicationandtheContactlessCommunicationAPI", 2008-10-24

[4] NFC Forum.(2004, march). Nokia, Philips and Sony Establish The Near Field Communication (NFC) Forum.

[5] [Online] developer.android.com. 